\title{
ARCHIVAR EN LA NUBE: REGLAS DE PRODUCCIÓN DEL DOCUMENTO CONTEMPORÁNEO. PARTE I: INDICADORES TECNOLÓGICOS
}

\author{
Alejandro Delgado-Gómez
}

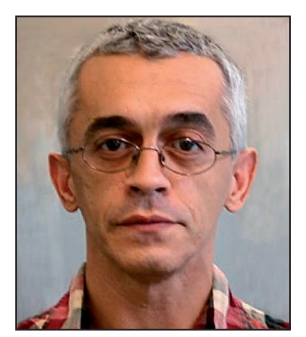

Alejandro Delgado-Gómez es técnico de archivos del Ayuntamiento de Cartagena y consultor senior de OdiloTID. Investigador afiliado del Center for Information as Evidence (Univ. of CaliforniaLos Angeles). Investigador asociado del Centre for Organisational and Social Informatics (Monash University). Colaborador del TEAM Catalonia de InterPARES 3 (University of British Columbia). Miembro del Observatorio Infoscopos (Universidad de Zaragoza). Miembro de los comités científicos de las revistas Tabula y Scire, y del Congreso Internacional Ibersid. Miembro del Grupo de Trabajo para la elaboración de las Normas Técnicas de Interoperabilidad del Esquema Nacional de Interoperabilidad y miembro de la Comisión Nacional de Normas de Descripción Archivística. Anteriormente ha desempeñado diversos puestos profesionales y en comités y proyectos de investigación nacionales e internacionales. Ha disertado y escrito ampliamente sobre archivos, gestión de documentos, bibliotecas y crítica de la cultura.

$$
\begin{array}{r}
\text { Archivo Municipal de Cartagena } \\
\text { Parque de Artillería. Plaza del General López Pinto, s/n. } \\
30201 \text { Cartagena } \\
\text { alejandro.delgado@ayto-cartagena.es }
\end{array}
$$

\section{Resumen}

Se parte de la aserción de que los actuales sistemas de conservación de documentos a largo plazo no son viables por motivos de escala y de coste. Con el fin de comprobarlo, se explora el actual entorno tecnológico de producción de documentos, con especial atención a los fenómenos de la distribución de sistemas, del cloud computing y de la web 2.0. En dos futuros artículos 1) se explorará el entorno social en que esta producción tiene lugar, con especial atención a la falta de interés por la memoria y por la petición de responsabilidades, y 2) se expondrá un potencial escenario de gestión de documentos que el archivero tendrá que abordar a corto plazo.

\section{Palabras clave}

Sistemas, Sistemas de gestión de documentos, Sistemas de información, Cloud computing, Web 2.0, Evidencia.

\section{Title: Archiving in the cloud: rules for the creation of contemporary records. Part I: Technologial indicators}

\begin{abstract}
Based on our assertion that current record preservation systems are not feasible in the long term for reasons of scale and cost, we explore the current technological environment where records are created, paying special attention to phenomena such as distributed systems, cloud computing and web 2.0. In two future articles 1) we will explore the social environment where this creation is performed, with special attention to the lack of interest in memory and accountability, and 2) we will suggest a potential scenario for recordkeeping that archivists will have to address at the short term.
\end{abstract}

\section{Keywords}

Systems, Recordkeeeping systems, Information systems, Cloud computing, Web 2.0, Evidence.

Delgado-Gómez, Alejandro. "Archivar en la nube: reglas de producción del documento contemporáneo. Parte I: Indicadores tecnológicos". El profesional de la información, 2011, julio-agosto, v. 20, n. 4, pp. 406-416.

http://dx.doi.org/10.3145/epi.2011.jul.07

\section{Introducción}

El presente es el primero de un conjunto de tres artículos que exploran la posibilidad de que un sistema de conservación de los documentos generados en un sistema de producción capaz de garantizar los requisitos de evidencia de tales documentos no sea posible ni deseable tal y como se concibe en la actualidad, y resulten precisos ciertos refinamientos.

El motivo es el hecho de que las actuales reglas de producción de documentos, que expresamos mediante la discusión 
de ciertos indicadores tecnológicos y sociales, no permiten concebir la conservación tal y como se concebía en la época del papel. Por motivos de espacio, en el presente texto exploramos sólo los indicadores tecnológicos; los indicadores sociales se expondrán en una segunda parte, y en una tercera se propondrá una reingeniería profesional tanto intelectual como práctica, derivada de los indicadores investigados en los dos primeros artículos, así como de la propuesta de un meta-OAIS integrador y orientado a los procesos.

Para proceder a tal exploración primero creemos necesario definir ciertos conceptos clave que aparecerán a lo largo de los textos.

\section{Documento}

En primer lugar, se suele entender por documento "una unidad indivisible de información constituida por un mensaje fijado a un soporte (registrado) de manera sintácticamente estable. Un documento tiene forma fija y contenido estable" (InterPARES 2). Además, se suele decir de un documento de archivo, que es "un documento realizado o recibido en el curso de una actividad práctica como instrumento o resultado de tal actividad, y acumulado para acción o referencia" (InterPARES 2). Por tanto, un documento de archivo se diferencia de otros documentos por el hecho de que constituye evidencia de acciones. En el presente texto utilizaremos únicamente el término "documento", entendido como sinónimo de "documento de archivo". Sin embargo, existen varios motivos por los que, en los nuevos espacios virtuales, las definiciones convencionales que acabamos de proporcionar no nos resultan útiles. Tres motivos clave son:

- Los documentos en espacios virtuales no tienen una forma fija ni un contenido estable, o al menos no existen garantías suficientes de que tales propiedades aparecerán y se mantendrán en todos los casos ni a lo largo del tiempo.

- Los documentos en espacios virtuales no pueden "acumularse" a posteriori, mucho tiempo después de que las actividades en las que se encuentran implicados hayan finalizado, porque entonces ya no pueden servir para acción ni referencia, o no pueden hacerlo con las suficientes garantías.

- La evidencia no tiene por qué estar contenida en documentos creados de acuerdo con criterios rígidos y, de hecho, en espacios virtuales que tienden a la proliferación y a la descentralización extremas, la evidencia puede estar contenida en cualquier ítem de información. Es decir, el concepto de documento derivado de la diplomática clásica no es útil en espacios virtuales, donde los componentes de la confiabilidad quedan desdibujados.

Por ello, delineamos una nueva definición de documento, basada, creemos, en suficiente garantía bibliográfica (por ejemplo, Ketelaar, 1999; Nesmith, 1999; McKemmish, 2005):

El documento es el resultado de la ejecución de procesos de comunicación de significado, significado que puede variar dependiendo de las circunstancias de la comunicación. En tanto resultado de un proceso de comunicación, es decir, de transacciones expresas entre dos o más partes, un documento es un tejido, un textus (Delgado-Gómez, 2010b).
En nuestra opinión, esta definición es lo suficientemente flexible como para poder ser utilizada de manera ventajosa en los siguientes sentidos:

- Acomoda las definiciones convencionales de documento y de documento de archivo proporcionadas en el párrafo precedente.

- Enfatiza la acción por encima del documento: el documento, como las propias definiciones convencionales admiten, es relativo con respecto a la acción, está subordinado a ésta.

- Permite expandir el universo de los documentos, al incluir documentos que no son el documento oficial generado durante el procedimiento administrativo derivado de las tradiciones jurídico-administrativas basadas en el Derecho Romano. El documento comporta evidencia, y ésta puede mostrarse en muchos soportes, no sólo en los expedientes de las burocracias latinas o de otros entornos (Reed, 2005).

- Permite desdibujar la férrea separación que históricamente forjaron las burocracias weberianas -de Max Weber, 1864-1920- (Bearman, 1994b), entre información y documento. Esta distinción deviene muy ambigua en espacios virtuales.

\section{Evidencia}

Un segundo término de relevancia es el de evidencia, a la que se suele definir como "todos los medios por los que cualquier hecho presunto, la verdad del cual está sometida a investigación, queda establecida o desmentida" (InterPARES 2). Se trata de una definición con un fuerte componente jurídico, que viene a equivaler a nuestro convencional valor probatorio o a la capacidad del documento para servir de testimonio. Sin embargo, en gestión de documentos, el concepto de evidencia es más amplio (Reed, 2005) e interdisciplinar (Twining, 2003; Furner, 2005), no se limita al ámbito jurídico.

Esta visión de la evidencia o del "ser testimonio de", se alinea mejor que la definición tradicional con la definición de documento que hemos propuesto. En el presente texto entenderemos por evidencia el hecho de que el documento es susceptible de servir como testimonio, con dos matices:

- Este testimonio no es exclusivamente jurídico-administrativo, sino también científico, cultural, personal, comunitario, etc.

- La evidencia puede estar contenida en muchos objetos y eventos, y el documento, en su sentido eurocéntrico, no ocupa una posición de privilegio.

Por tanto, el documento no se produce en condiciones de abstracción, sino siempre en entornos socio-culturales, económicos, disciplinares, etc., dados. Esta visión de la evidencia permite considerar que ésta se encuentra registrada en multitud de formas, formatos o soportes, no sólo en el documento oficial: un sms, una conversación por chat, la recogida de datos científicos, una performance, un recuerdo registrado en la memoria individual, un ritual funerario, un post en un blog, la cinética de los festejos indígenas, un monumento, una ciudad, etc., comportan evidencia de acciones $\mathrm{y}$, en ese sentido, son documentos. 
El hecho de que los objetos o eventos que tenemos entre manos sean o no documentos es en realidad irrelevante: lo importante es que tales objetos o eventos sean evidencia de acciones y que estén registrados como información con ciertas garantías jurídicas o sociales. Es decir, la separación jurídica convencional en nuestra tradición entre documento, testimonio oral y objeto no es aplicable a la gestión de documentos: el documento, el testimonio oral y el objeto están subordinados al ser evidencia de acciones. Este ser evidencia de acciones no les viene dado en esencia, sino que depende de las circunstancias en un contexto dado.

La evidencia tiene dos aplicaciones básicas: la responsabilidad (la petición de cuentas, en nuestra tradición) y la memoria (el valor para la investigación, en nuestra tradición). Preferimos el uso de los términos responsabilidad y memoria, porque nos parecen más abarcadores $y$, en cualquier caso, nunca constituyen compartimentos estancos, sino que siempre están entrelazados de múltiples maneras (DelgadoGómez, 2007).

Los conceptos responsabilidad y memoria no constituyen compartimentos estancos, sino que siempre están entrelazados de múltiples maneras

\section{Sistemas de conservación y de producción}

Otras dos expresiones que aparecen en los textos con frecuencia son "sistema de conservación" y "sistema de producción". La primera de ellas está bien consolidada en archivística: un sistema de conservación es "un conjunto de reglas que gobiernan el mantenimiento permanente intelectual y físico de los documentos adquiridos y las herramientas y los mecanismos utilizados para implantar esas reglas" (InterPARES 2). Es decir, un sistema de conservación viene a equivaler a lo que en nuestra tradición se conoce como archivo permanente, histórico, definitivo, etc.

La expresión "sistema de producción" significa un "conjunto particular de actividades (sistema de manejo) desarrolladas para producir una serie definida de productos o beneficios" (FAO, 1997); es decir, el sistema de producción no es otra cosa que el entorno de gestión en el que se producen documentos que reflejan acciones. Una vez finalizadas éstas, los documentos se transfieren al sistema de conservación o archivo.

Como se indicó al comienzo, es perspectiva del presente artículo el que esta separación entre el sistema de conservación y el sistema de producción no es ni viable ni deseable en espacios virtuales, puesto que se pierde el valor de evidencia de los documentos; es decir, estos sistemas no deben mantenerse separados, sino en interrelación.

El concepto de regla de producción se corresponde con las acepciones dos y cuatro del Diccionario de la Lengua de la Real Academia Española: "Aquello que ha de cumplirse por estar así convenido por una colectividad" y "Estatuto, constitución o modo de ejecutar algo" (Real Academia Española). Es decir, en primer lugar han de producirse documentos, porque así se ha acordado en un contexto dado; pero, en segundo lugar, deben producirse de un modo determinado. Este concepto puede entenderse de manera estricta, por ejemplo regla como equivalente de procedimiento administrativo, de proceso de trabajo, de flujo de tareas, etc.; pero también puede entenderse de manera general o global: las comunidades o las sociedades tienen reglas explícitas o tácitas de producción de documentos que revisten carácter social, político, jurídico-administrativo, científico, etc. (Foucault, 1999); y también revisten carácter tecnológico (Derrida, 1996; Ketelaar, 2007; Delgado-Gómez, 2009). Por expresarlo de manera breve, las reglas de producción del documento son aquellas convenciones sociales y tecnológicas que deciden qué documentos pueden o deben crearse, o no pueden ni deben crearse, en un contexto dado, porque son o no son necesarios, oportunos, posibles, o por cualquier otro motivo (Delgado-Gómez, 2010). Este concepto ha de matizarse en dos sentidos:

En primer lugar, el hecho de que existan reglas de producción no significa que no puedan producirse documentos que no cumplan tales reglas, pero serán documentos que estarán fuera del orden establecido. El hecho de que no cumplan las reglas no los anula como documentos, pero no están en el orden del discurso de una comunidad dada.

En segundo lugar, del hecho de que aparentemente no existan reglas de producción no se sigue que no existan. Más bien, las reglas de producción son a menudo tácitas o el entorno tiende a facilitar su olvido; pero incluso el aparente "todo vale" de los actuales espacios virtuales es una regla de producción.

\section{Espaciotiempo}

Finalmente, en el presente texto se utiliza el término "espaciotiempo" en el mismo sentido que en el paradigma del continuo de los documentos: "En física, espaciotiempo se refiere a 'cualquier modelo matemático que combina espacio y tiempo en un solo continuo' con cuatro dimensiones -tres de espacio y uno de tiempo: 'los espaciotiempos son las áreas en las que todos los eventos físicos tienen lugarun evento es un punto del espaciotiempo especificado por su tiempo y por su lugar'"' (McKemmish, 2010).

\section{Un sistema es en realidad un conjunto de sistemas locales o remotos, que se conectan de manera puntual o perma- nente, para producir una transacción y la información asociada a la misma}

Nos apropiamos del concepto por el siguiente motivo: las acciones de las que los documentos son reflejo no suceden en abstracto, sino que están situadas en un lugar y en un momento, son relativas a las circunstancias de su ocurrencia (Foucault, 2005). Conocer este lugar y este momento es imprescindible para comprender las circunstancias en las que el documento ocurre (Hurley, 2001-2004). No es función del archivero determinar sus valores de verdad ni éticos, sino comprenderlos en tales circunstancias y proporcionar y mantener una explicación adecuada de las mismas. 


\section{Entorno tecnológico de producción del documento contemporáneo}

\section{Primer indicador: carácter transaccional y contextual del documento}

Los documentos son por naturaleza transaccionales y, en el caso de los electrónicos, no pueden ser objeto de transferencia entre sistemas, puesto que en este caso pierden su vínculo inmediato con la transacción de la que son reflejo (Bearman, 2010). A esta aserción añadimos que los documentos son también por naturaleza contextuales (McKemmish, 2001), y una transferencia sin información adicional también los separa, en principio, de su contexto de producción, quedando comprometido por tanto su carácter de ser evidencia de acciones.

El hecho de que un sistema de conservación funcione de manera separada de un sistema de producción siempre pone en peligro el carácter de evidencia de acciones del documento, una vez que pasa de un sistema a otro. La única situación ideal es aquella en la que el sistema de producción es lo suficientemente seguro y está sujeto a tantos controles que puede funcionar simultáneamente como sistema de conservación. El problema es el hecho de que los sistemas de producción suelen ser extremadamente inestables y desregulados (ISO 18492; Nesmith, 2002), de tal modo que resultan poco fiables como sistemas de conservación. Por tanto, se precisa controlar y documentar el modo en que se genera el documento, y se precisa que esta documentación acompañe al documento, en forma de metadatos, durante toda su vida.

El mejor ejemplo en un entorno analógico es el de los sistemas de registro de origen prusiano (Gilliland-Swetland, 2000; Cook, 2001). En un entorno digital, la clonación de tales sistemas de registro constituiría una excelente solución (Reed, 2005; Bearman, 2010). El motivo es el siguiente: los sistemas de registro mencionados no sólo daban testimonio de que el documento había entrado en el sistema, sino que realizaban el seguimiento del mismo durante toda su vida, es decir, lo controlaban asignándole metadatos. En un entorno analógico, estos metadatos quedan vinculados de manera inextricable al documento y su alteración se detecta con facilidad; en un entorno digital, los metadatos pueden quedar vinculados de manera inextricable o no, pero su alteración se detecta con mayor dificultad.

\section{Segundo indicador: inexistencia del documento elec- trónico}

El motivo por el que las acciones que se realizan en entornos digitales producen mayor preocupación y deberían ser objeto de un escrutinio minucioso se ha explicado hasta la saciedad (por ejemplo, Heslop; Davis; Wilson, 2002; desde otra perspectiva, Duranti; Thibodeau, 2006), pero quizá convenga recordarlo. Aunque los documentos analógicos y los electrónicos cumplen la misma funcionalidad, el ser evidencia de acciones, la naturaleza de uno y de otro es muy diferente, puesto que el primero tiene, como se dijo, una forma fija y un contenido estable, mientras que el segundo es un conjunto desagregado y distribuido de datos e instrucciones (lacovino, 2010), que se recomponen o agregan bajo demanda para generar en pantalla una manifestación o instanciación de aquello que esperamos ver. Es decir, en sentido estricto un documento electrónico no existe, simplemente se manifiesta, y deja de hacerlo cuando se cierra la sesión o se apaga el ordenador. La figura 1 quizá represente con mayor claridad la naturaleza desagregada del documento electrónico.

En sentido estricto un documento electrónico no existe, simplemente se manifiesta, y deja de hacerlo cuando se cierra la sesión o se apaga el ordenador

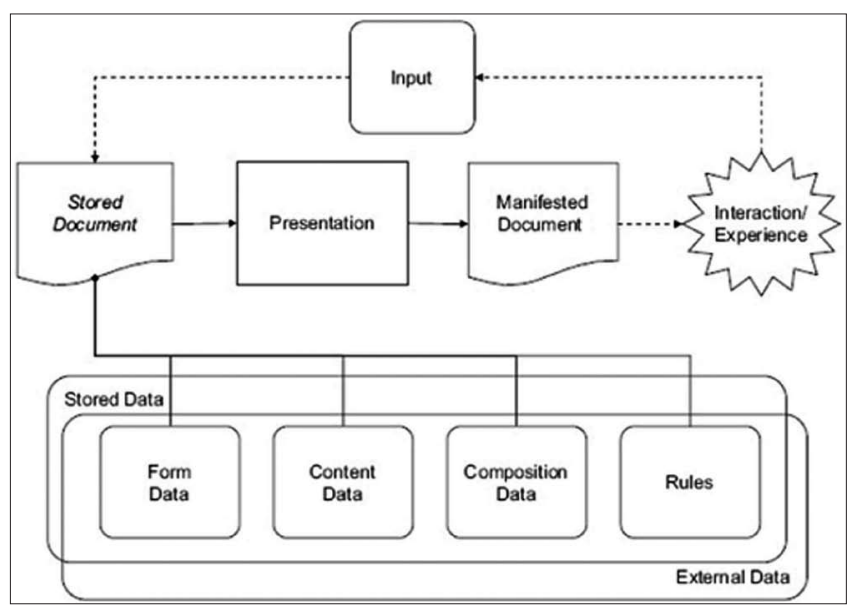

Figura 1. Modelo genérico de documentos almacenados y manifestados (Duranti; Thibodeau, 2006). Reproducido con permiso.

Por ejemplo, un documento redactado con el procesador de textos Microsoft Word "parece" un documento cuando se muestra en pantalla, pero dentro del ordenador no existe nada similar a ese documento con negritas, sangrías, etc. Existen bibliotecas de vínculos dinámicos, datos, reglas de negocio, conexiones a una base de datos Access, enlaces a un fichero de imagen gif, etc. Estos componentes ni siquiera están almacenados en el mismo lugar dentro de un ordenador, puesto que dentro de un ordenador el orden de almacenamiento es aleatorio (Bearman, 1994a). De manera cada vez más frecuente en entornos distribuidos ni siquiera están en el mismo ordenador. Lo único fácilmente reconocible en pantalla es un icono. Si se hace doble clic sobre él, todos aquellos componentes serán invocados para que se muestre la apariencia de documento que esperamos ver (Delgado-Gómez, 2010a).

La legislación, por ejemplo, la Ley 11/2007, de 22 de junio, de Acceso electrónico de los ciudadanos a los servicios públicos; el Real decreto 1671/2009, de 6 de noviembre, por el que se desarrolla parcialmente la citada Ley 11/2007; el Real decreto 3/2010, de 8 de enero, por el que se regula el Esquema Nacional de Seguridad en el ámbito de la Administración Electrónica; o el Real decreto 4/2010, de 8 de enero, por el que se regula el Esquema Nacional de Interoperabilidad en el ámbito de la Administración Electrónica hacen referencia a conceptos como el de documento electrónico o el de expediente electrónico. 
Somos de la opinión de que los últimos desarrollos legislativos nacionales sobre administración electrónica son muy avanzados, aunque muestran una fuerte y probablemente inevitable dependencia de la legislación tradicional que constituye su raíz, a saber, la Ley 30/1992, de 26 de noviembre, de Régimen Jurídico de las Administraciones Públicas y del Procedimiento Administrativo Común. Pero también somos de la opinión de que esta dependencia tiene un inconsciente carácter positivo, al facilitar una transición sin traumas desde un paradigma familiar a otro por completo diferente y cuya lógica se distancia de la lógica humana.

Dos debilidades de la legislación actual sobre administración electrónica, en lo que concierne al archivo, tienen que ver con la traslación de nociones clásicas al entorno digital:

- El concepto de ciclo de vida de la información no es viable en un medio en el que la información tiende a crearse, gestionarse, utilizarse y reciclarse de manera continua, no discreta (Schauder; Stillman; Johanson, 2004; 2005). Una representación del modo en que funciona la información en entornos digitales puede observarse en la figura 2.

- El concepto de conservación a largo plazo en un contexto tecnológico extremadamente cambiante tampoco es viable, siendo preferible recurrir al concepto de conservación durante muchos períodos breves, como método más eficaz (Ross; Hedstrom, 2003).

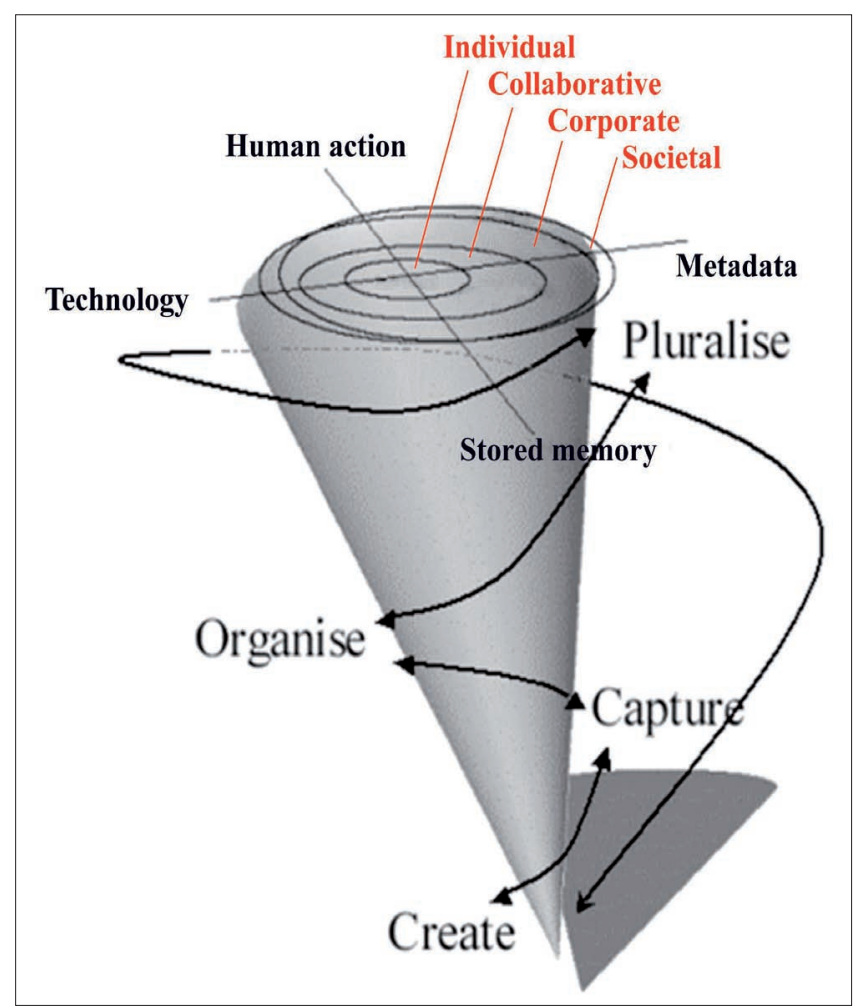

Figura 2. Visualización dinámica del modelo continuo de la información (Schauder; Stillman; Johanson, 2005). Reproducido con permiso.

En cualquier caso, entre el año 2007 y el año 2010, la legislación ha ido liberándose de matices propios de la era papel, y es de prever que en algún momento del futuro promulgue abiertamente que no se puede pensar de los documentos electrónicos "con mentes de papel" (Cook, 1994). En este sentido, resulta esperanzador destacar el hecho de que la legislación haya ido distanciándose de la orientación hacia la tecnología (las leyes de Protección de datos o de Firma electrónica son cuestionables en la medida en que se redactan en términos que no tienen en cuenta el imparable cambio tecnológico), para aproximarse a una orientación a los procesos, particularmente, por ejemplo, en el Esquema Nacional de Interoperabilidad.

La reutilización de la información en diferentes espacio-tiempos permite la realización de multiversos archivísticos que obligan a replantear qué cosa sea un archivo

\section{Tercer indicador: el documento es el sistema y el ítem}

El anterior ejemplo basado en Word es una muestra bastante simple de lo que sucede cuando se usa un software de escritorio; pero los ámbitos en que tiene lugar esa manifestación del documento ya no están aislados, sino que son de manera creciente invisibles, invasivos, inestables, dinámicos, interactivos, distribuidos, reutilizables y multipropósito (Delgado-Gómez, 2010a). El conocimiento exhaustivo de tales ámbitos y la documentación de los mismos resultan necesarios para que el documento electrónico siga siendo evidencia de acciones. De manera breve: si separamos algunos datos con apariencia de documento del complejo entramado en el que se produjeron no tendremos toda la historia de las circunstancias de su producción y, por tanto, quizá tengan valor informativo, pero carecerán de valor de evidencia, salvo en el supuesto de que tales datos vengan acompañados de una exhaustiva documentación, y de que esta documentación sea admisible en el entorno regulador de un espaciotiempo dado. En este sentido, en el ámbito de la gestión de documentos electrónicos diríamos que el documento es el propio sistema.

De nuevo, esto también sucede con el papel, aunque el ejercicio de control en el mismo es más sencillo. No obstante, la mayor parte de las tradiciones burocráticas no han permitido a los archiveros ejercer este control, con el beneplácito a veces de ellos mismos, que no han dudado en aceptar el jenkinsoniano principio de santidad de la evidencia. Sin embargo, ya desde el momento en que se enunció el principio existían pruebas de que las burocracias no son santas y precisan ser controladas: mientras Sir Hilary Jenkinson enunciaba su conocido principio, sobre la base de que todos sus colegas en todos los ministerios británicos eran honestos caballeros eduardianos, su propio equipo de trabajo alteraba y destruía los documentos bajo su custodia para ocultar el mal hacer del Alto Mando durante la Primera Guerra Mundial (Delgado-Gómez, 2010b).

Los modernos sistemas que producen documentos exceden la escala humana, precisamente por las características de los mismos que enunciábamos en el párrafo precedente; pero el problema no es sólo el volumen de información, sino más bien el modo extremadamente desconcentrado, descentra- 
lizado, auto-regulado y totalizador en que se produce ésta (Floridi, 2009a, 2009b). La diferencia con respecto a otras inflaciones informativas a lo largo de la historia es que en esos casos la información o los documentos se producían en un número reducido de nodos, conocidos y controlados, al contrario de lo que sucede hoy en día. De manera creciente, un sistema es en realidad un conjunto de sistemas locales o remotos, que se conectan de manera puntual o permanente, para producir una transacción y la información asociada a la misma. Estas conexiones, a mayor abundancia, son ellas mismas transacciones que precisan de reflejo estable.

Además, tales sistemas, en muchos casos, no han sido concebidos como sistemas "puros" de gestión de documentos; de hecho, su funcionalidad no es esa: los numerosos sistemas de gestión de las organizaciones han sido concebidos para gestionar la contabilidad, los recursos humanos, o la georreferencia policial, pero no para producir documentos. Básicamente, en tales ensamblajes de sistemas se produce información y se generan datos que son susceptibles de entrar en conexión para generar documentos, o la apariencia de tales, en la medida en que estas conexiones son transacciones y tienen un reflejo en forma de evidencia, pero las posibilidades de estabilizar esa evidencia queda minimizada (DelgadoGómez; Rodríguez-Gutiérrez; Tornel-Cobacho, 2009). Los modernos sistemas o entramados de sistemas organizativos pueden actualizar la información y generar nuevos datos en cuestión de nanosegundos, lo cual hace realmente complicado el control de los mismos. No obstante, y al mismo tiempo que el documento es el sistema, el documento también es cada ítem de datos sobre los que se debe ejercer control a efectos de conservación de la evidencia.

\section{Cuarto indicador: la información ocupa lugar}

Esta nueva situación, que pone ya en riesgo las posibilidades de garantizar evidencia en el ámbito organizativo, alcanza dimensiones desmesuradas si tomamos en consideración otros ámbitos, u otras actividades dentro del propio ámbito organizativo. Por ejemplo, las instituciones se han lanzado a una carrera desenfrenada para liberar sus contenidos y ponerlos a disposición de la ciudadanía sobre la Red (Pares, Hispana, Europeana, son sólo ejemplos populares), lo cual es altamente loable y democrático, pero también altamente costoso: servidores de bases de datos, servidores de ficheros, contratación de ancho de banda, soportes para realizar copias de seguridad, costes de la energía eléctrica necesaria o personal de mantenimiento.

Brown (2010) ha hecho notar la circunstancia de que "la producción de información digital ya ha superado la capacidad global de servidor en un factor estimado de 4 ó 5", es decir, en términos matemáticos, estaríamos produciendo más de un $400 \%$ más de información de la que somos capaces de almacenar. Sin embargo, también indica que éste no es el mayor problema, puesto que la ingeniería de almacenamiento se está desarrollando cada vez con menores costes. El problema, por tanto, no es disponer de almacenes digitales, sino de administrar, gestionar, mantener éstos de manera sostenible. De igual modo que lo preocupante no es el volumen de información, sino el modo en que se produce, en este caso lo preocupante tampoco es el volumen, sino los medios para gestionarlo.
Esto no sería demasiado relevante si la función de garantía de evidencia y la función de difusión de contenidos constituyeran compartimentos estancos dentro de una organización; sin embargo, en la gestión de documentos contemporánea, ambas funciones tienden a ser continuas o, al menos, a desenvolverse bajo un solo paraguas presupuestario; es decir, se suelen enmarcar dentro de un solo sistema o entramado de sistemas, lo cual significa que deben compartir recursos, técnicos y económicos, en un momento en que tales recursos no son precisamente un bien copioso. Además, esta democratización de los contenidos de las organizaciones contribuye a la sobreabundancia de información que ya las propias organizaciones, en sus actividades relacionadas con la evidencia, generan.

Por poner un ejemplo, durante la administración Bush se transfirieron a los National Archives and Records Administration (NARA) de los EUA 270.000.000 de objetos digitales que constituían un total de 72 terabytes. Estas cifras asustan, pero asustan aún más si se comparan con los algo más de 30.000.000 de objetos digitales que se transfirieron durante la administración Clinton (Thibodeau, 2009), y que hablan de un incremento descontrolado y de una proliferación de datos cuya sostenibilidad bien pudiera ponerse en cuestión. El autor proporciona estas cifras como indicadores de éxito; no obstante, es el punto de vista del presente texto el que constituyen indicios de un notable fracaso: NARA está gastando dinero, tiempo de personal y recursos informáticos en almacenar y mantener 72 terabytes de información que en un momento no determinado del futuro carecerán de valor de evidencia.

\section{A pesar de la vigilancia y de la seguridad de determinadas nubes privadas, existe un margen de inseguridad que las orga- nizaciones tendrán que abordar}

El motivo es muy simple: dado que no es previsible que las tecnologías se detengan, los datos tienen que convertirse y migrarse de manera continua. Cada uno de estos movimientos provoca cambios perceptibles o imperceptibles (Harvey, 2010), de tal modo que en algún punto no determinado del futuro, el hecho de que se diga de un documento que es auténtico, que es lo que pretendía ser en el momento en que fue creado, carecerá de sentido, porque su estructura se habrá alterado hasta límites que en la actualidad consideramos como inaceptables. Además, no existen plenas garantías de que en uno o varios de estos pasos no se produzcan pérdidas de datos de contexto y de contenido (Rothenberg, 2000; Ketelaar, 2010).

\section{Quinto indicador: la democratización de internet}

Fuera del ámbito de las organizaciones, la democratización de internet -es decir, el uso de redes sociales, blogs, wikis, sindicación de contenidos, depósitos colectivos de documentos, vídeos o imágenes, etc.-, ha permitido que se produzcan y se conozcan documentos no oficiales que o complementan o discuten a los documentos oficiales. Esto es intelectualmente beneficioso, pero crea a su vez nuevos 
problemas. El principal beneficio del uso de tales instrumentos es la posibilidad de que el documento pueda atravesar espaciotiempos diferentes y ser usado de manera diferente en cada uno de ellos, con un significado diferente del significado original.

Como contrapartida, se vuelve a producir una inflación de información cuya gestión el usuario final delega en corporaciones de mucho más amplio alcance que nuestras organizaciones convencionales, a partir de la asunción de que estas grandes corporaciones llevarán a cabo una buena gestión de documentos, o, en el peor de los casos, sin que el usuario final sienta el menor interés acerca del modo en que se gestionan sus documentos, comprometiendo de este modo nociones hasta ahora consideradas prácticamente inviolables, como las de privacidad, intimidad, o identidad (lacovino, 2007, 2010). Es decir, el usuario final prefiere depositar sus documentos en Google, Yahoo o Facebook, antes que en un archivo que esté bajo su control.

El control sobre un nodo de la red no implica necesariamente el control sobre otros nodos con los que aquél puede mantener relaciones puntuales o estables

En lo que concierne a nuestras organizaciones, este fenómeno tiene ciertas consecuencias:

- En primer lugar, la reutilización de la información en diferentes espaciotiempos permite la realización de multiver$\operatorname{sos}^{1}$ archivísticos que obligan a replantear qué cosa sea un archivo, o, en términos de McKemmish (2010), que desplazan el centro de interés desde el archivar blogs hacia el uso del blogging como máquina de archivar.

- En segundo lugar, esta nueva economía social en la que se mantiene la creencia de que el documento es gratuito y se puede generar hasta el infinito, también obliga a repensar la noción de valoración y quién llevará a cabo ésta. En este escenario queda excluido el momento de la archivalización que propusiera Eric Ketelaar (1999), puesto que no existe límite para que todo sea documento. También queda excluido el momento de la archivación ${ }^{2}$, puesto que la gestión del documento creado es irrelevante. Así pues, el archivar los documentos de la sociedad se convierte en una tarea ad infinitum en manos de corporaciones con intereses económicos más o menos explícitos. Esto no es necesariamente malo: esos mismos intereses económicos deberían ser motivo suficiente para prestar un buen servicios a sus usuarios. Pero tampoco es necesariamente bueno, siendo la desagregación de datos y la pérdida de la noción de individuo los elementos negativos más relevantes.

- En tercer lugar, las propias organizaciones están ya utilizando estos instrumentos con diferentes fines (Samouelian, 2009). Si bien no es frecuente que uno de estos fines sea la toma de decisiones oficiales, se puede argumentar que la facilidad de uso y la flexibilidad de los mismos motivará a las organizaciones y a los individuos que las componen a utilizar tales mecanismos de manera cada vez más profunda.
- En cuarto lugar, si grandes corporaciones ofrecen al usuario mecanismos de producción, de gestión y de conservación mucho más ágiles que los ofrecidos por las burocracias, las organizaciones burocráticas tendrán que comenzar a asemejarse a, y a negociar con, tales corporaciones, que mantienen mucha más información que la que puedan contener cada una de las organizaciones por separado. De hecho, ya han comenzado a negociar con ellas, tal y como sugerirían ejemplos como las ventas de datos de disidentes a gobiernos dictatoriales, la censura de Google por parte del Gobierno chino, o la guerra entre organizaciones gubernamentales y Google, ante el intento de esta última corporación de hacerse con el monopolio de la distribución del libro digital.

\section{Sexto indicador: la pérdida del control}

Si el mantenimiento de los grandes sistemas o conglomerados de sistemas que permiten que las organizaciones funcionen es caro en un momento de crisis y si el funcionamiento de tales organizaciones implica la generación de millones de datos cuyo almacenamiento también es caro e inmanejable, el modo de provisión de servicios conocido como cloud computing ${ }^{3}$, que es barato, ecológico, ahorra energía, gastos de personal, etc., se configura como una opción bastante seria de futuro (Gilder, 2006; Cloud Computing Use Case Discussion Group, 2009; Mell; Grance; 2009; Spinola, 2009). Es cierto que, junto a sus ventajas, también se han citado frecuentemente muchos de sus riesgos (Council of Australasian Archives and Records Authorities, 2010; Gellman, 2009): diferentes legislaciones sobre privacidad, posible pérdida de datos, posibles suplantaciones de identidad, posibles accesos no autorizados, copias redundantes no controladas, etc.

No obstante, también es cierto que existen muchos modos de utilizar servicios de cloud computing, y la nube no tiene por qué ser forzosamente pública. Por ejemplo, la Red SARA no dejaría de ser una nube, en este caso cerrada y segura, para la realización de comunicaciones entre administraciones; las administraciones japonesa y estadounidense ya han lanzado su estrategia de prestación de servicios cloud (Chan, 2009; Foley, 2009), y la administración británica también se está preparando para ello. Por supuesto, a la luz de las recomendaciones que se están elaborando para afrontar los riesgos del cloud computing, no podemos dejar de plantear el hecho de que incluso las nubes seguras son inseguras, de tal modo que colocar servicios, sistemas o depósitos en la nube implicará una continuada tarea de gestión del riesgo y de la incertidumbre, que no siempre será capaz de transformar esas incertidumbres en certezas.

El riesgo es inevitable, y todas las organizaciones tienen que emprender acciones para gestionar el riesgo de modo que se pueda justificar hasta un nivel que sea tolerable. La cantidad de riesgo de la que se juzga que es tolerable y justificable es la 'apetencia del riesgo' (Drambora, 2007).

De manera breve: a pesar de las medidas de vigilancia y de seguridad de determinadas nubes privadas, existe un margen de inseguridad que las organizaciones tendrán que abordar, por la misma naturaleza altamente descentralizada de las redes contemporáneas, en las que el control sobre un 
nodo de la red no implica necesariamente el control sobre otros nodos con los que aquél puede mantener relaciones puntuales o estables.

Por ejemplo, si una administración que funciona bajo el paraguas de la Red SARA externaliza alguno de sus servicios, incluso mediante un contrato en el que se especifican las mayores medidas posibles de seguridad, ¿qué posición ocupa el nodo del proveedor de servicio? ¿Está dentro de la Red $S A R A$ ? ¿Está fuera? ¿A qué otras organizaciones presta servicios ese proveedor? En cualquiera de los casos, ya existe una brecha. La seguridad en la red es una cuestión de grado, y ni la mejor legislación ni las mejores normas existentes son capaces de asegurar que es imposible el que tales nodos débiles no se generarán. Antes al contrario, la privatización y la externalización de servicios, dado una vez más el fenómeno de la crisis económica, tenderán a incrementarse (lacovino, 2010).

Ni siquiera es necesario salir del ámbito organizativo: mientras se redacta el presente artículo aparece en la prensa regional la noticia de que una de las oficinas del organismo de empleo SEF ha estado vendiendo datos personales de desempleados a bancos que querían conocer su situación financiera. El valor de estos datos personales se cifraba entre veinte y cuarenta euros (Fernández, 01/03/2011). El mal hacer público se examinará en un artículo posterior, pero parece configurarse como peligroso enemigo de las medidas de seguridad.

\section{El principal indicio que debe tomarse en cuenta es el creciente desinterés ciu- dadano por la noción de evidencia, en cualquiera de sus dos funcionalidades, la responsabilidad y la memoria}

\section{Séptimo indicador: comunicaciones débiles y nuevos actores}

Entendemos por comunicación débil de datos aquella que se produce mediante móviles, portátiles, iPods, wiis, PDAs, y otros dispositivos que no están bajo el control físico y estricto de una organización (Ketelaar, 2006). Algunos ordenadores de escritorio van siendo sustituidos por dispositivos móviles y el que la transmisión de información organizativa, incluida la toma de decisiones, se produzca desde tales aparatos será un hecho cada vez más frecuente. Dada su facilidad de uso, su portabilidad y sus amplias posibilidades, no es previsible que estos usos se detengan. Por ejemplo, es normal el hecho de que un ejecutivo dé una orden a través de su portátil o de su Blackberry; pero pensemos que ese mismo ejecutivo está en casa, jugando con sus hijos, y surge una urgencia en el trabajo. ¿Por qué habría de dejar a sus hijos, encender el ordenador y enviar su decisión, si puede hacerlo a través de la Wii con la que está jugando? En efecto, este tipo de mecanismos se están utilizando mayoritariamente para disfrutar del ocio, pero con las debidas conexiones, una pantalla de televisión y un mando inalámbrico que apenas requiere movimiento físico un anciano podría pedir recetas a su médico sin moverse del sofá, un estudiante podría rea- lizar un examen, una persona disminuida podría alertar de una crisis, y un consejo ejecutivo podría tener lugar a través de miis o avatares de cualquier otro tipo. Esto último, incidentalmente, dejaría la puerta abierta a una discusión acerca del problema de las ciber-identidades.

En este sentido, no es casual el reciente interés por el establecimiento y la consolidación de relaciones con la ciencia forense digital (Duranti, 2009), cuyo territorio es el de estas comunicaciones fugaces en las que la determinación de las condiciones de autenticidad no depende de nosotros, sino de la máquina. Esta nueva alianza, no obstante, obliga a reflexionar acerca de si nuestro rol en sociedad es útil. En efecto, si la ciencia forense digital es capaz de proporcionar mejor evidencia que la alojada en nuestros sistemas de conservación y es capaz de estabilizarla en grado suficiente y fiable, siquiera en un plazo corto, nuestros sistemas de conservación devienen irrelevantes o, en el mejor de los casos, evidencia de segundo orden, cuya finalidad es únicamente servir de apoyo a la evidencia de primer orden proporcionada por la bitácora de un móvil o el seguimiento de la ruta de una red pederástica desde el ordenador de un cliente hasta el servidor que aloja las imágenes. En relación con la ciencia forense digital, también deberíamos aprender algo de disciplinas que son capaces de capturar evidencia a medida que se va produciendo, no a posteriori, cuando ya carece de valor.

En definitiva, gestionar sistemas se convertirá en el proceso de negociar con máquinas que negocian además con otras máquinas en un espaciotiempo dado, y que a su vez negocian con máquinas diferentes y remotas que negocian con otros humanos en espaciotiempos que no nos pertenecen (Delgado-Gómez, 2010a). En este escenario es donde el archivero, según todos los indicios, tendrá que gestionar los objetos susceptibles de comportar evidencia en un futuro próximo (McKemmish, 2010).

\section{Notas}

1. El término "multiverso" también se utiliza en el presente texto en el sentido en el que se utiliza dentro del paradigma del continuo de los documentos: "De acuerdo con el Oxford English Dictionary, el término multiverso fue acuñado originalmente en 1895 por el psicólogo William James, y se utiliza ahora para referirse a la serie hipotética de múltiples universos posibles. Ha sido explorado en el contexto de muchas disciplinas diferentes, incluidas la cosmología, la física, la astronomía, la psicología y la literatura.

http://en.wikipedia.org/wiki/Multiverse

2. En el presente texto se entiende la archivalización como el momento en el que, consciente o inconscientemente, y a nivel cultural, organizativo o personal, se decide que merece la pena convertir algo en documento; la archivación, como el momento en que algo es inscrito como documento; y el archivar, en sentido estricto, como el momento en que el documento así inscrito se conserva como valioso (Ketelaar, 1999).

3. La definición convencional de cloud computing diría que es "un modelo para hacer posible un acceso conveniente y bajo demanda, a través de las redes, a un depósito compartido de recursos informáticos configurables (p. ej., redes, 
servidores, almacenamiento, aplicaciones y servicios) que pueden usarse y desecharse rápidamente con un mínimo esfuerzo de gestión y de interacción por parte del proveedor de servicios" (Mell; Grance, 2009).

\section{Agradecimientos}

El autor desea agradecer los comentarios de Alfonso DíazRodríguez, Joaquim Llansó-Sanjuán y Cayetano Tornel-Cobacho, así como los de los dos revisores anónimos, que han contribuido extremadamente a mejorar el artículo.

\section{Referencias}

Bearman, David. "Architectural strategies for records capture". En: Seminario internacional o futuro da memoria: o patrimonio arquivístico dixital. Santiago de Compostela, 1819 de nov. de 2010.

Bearman, David. "Archival principles and the electronic office". Electronic evidence: strategies for managing records in contemporary organizations. Pittsburgh: Archives \& Museums Informatics, 1994a.

Bearman, David. "Diplomatics, Weberian bureaucracy, and the management of electronic records in Europe and America". Bearman, David: Electronic evidence: strategies for managing records in contemporary organizations. Pittsburgh: Archives \& Museum Informatics, 1994b, pp. 254-277.

Bearman, David. "Documenting documentation". Archivaria, 1992, n. 34, Summer, pp. 33-49.

Bearman, David. "Item level control and electronic recordkeeping". Archives \& museum informatics, 1996, v. 10, n. 3, pp. 195-245.

http://www.archimuse.com/papers/nhprc/item-lvl.html

Bearman, David. "Moments of risk: identifying threats to electronic records". Archivaria, 2006, n. 62, Fall, pp. 15-46.

Brown, Richard. "La macrovaloración en el siglo veintiuno: hacia un nuevo marco documental para la memoria pública". En: Seminario internacional o futuro da memoria: o patrimonio arquivístico dixital. Santiago de Compostela, 18-19 de nov. de 2010.

Chan, Tony. "Japan to build massive cloud infrastructure for e-government". En: Greentelecomlive, 2009.

http://www.greentelecomlive.com/2009/05/13/japan-tobuild-massive-cloud-infrastructure-for-e-government

Cloud Computing Use Case Discussion Group. Cloud computing use cases white paper. Version 2.0, 2009.

http://www.scribd.com/doc/18172802/Cloud-ComputingUse-Cases-Whitepaper

Cook, Terry. "Archival science and postmodernism: new formulations for old concepts". Archival science, 2001, v. 1, n. 1, March, pp. 3-24.

Cook, Terry. "Electronic records, paper minds: the revolution in information management and archives in the postcustodial and post-modernist era". Archives and social studies: a journal of interdisciplinary research, 1994, v. 1, n. 0. http://socialstudies.cartagena.es/images/PDF/no0/cook_ electronic.pdf
Council of Australasian Archives and Records Authorities. Advice on managing the recordkeeping risks associated with cloud computing. Australasian Digital Recordkeeping Initiative, 2010.

Derrida, Jacques. Mal de archivo: una impresión freudiana. Madrid: Trotta, 1996.

Delgado-Gómez, Alejandro. El centro y la equis: una introducción a la descripción archivística contemporánea. Cartagena: Ayuntamiento: 3000 Informática, 2007.

Delgado-Gómez, Alejandro. “La redefinición del trabajo: tecnologizamos nuestra práctica o la tecnología decide nuestro ejercicio profesional". Tabula, 2009, n. 12, pp. 103-114.

Delgado-Gómez, Alejandro. Las reglas de producción del documento en los entornos digitales contemporáneos: aspectos teóricos y estudio de caso de implantación práctica, 2010a. http://eprints.rclis.org/18612

Delgado-Gómez, Alejandro. "Los archivos como construcción social". El archivo como construcción social. Tenerife: Anroart, 2010b, pp. 7-112.

Delgado-Gómez, Alejandro. “Documentos y poder: órdenes del discurso". Anales de documentación, 2010c, v. 13, pp. 117-133.0

http://revistas.um.es/analesdoc/article/view/107051

Delgado-Gómez, Alejandro; Rodríguez-Gutiérrez, Miguel; Tornel-Cobacho, Cayetano. "El desarrollo de un sistema de gestión de expedientes mediante estrategias interdisciplinares: el caso del Ayuntamiento de Cartagena". En: Fesabid'09: XI Jornadas españolas de documentación. Zaragoza, 20-22 de mayo 2009, pp. 307-314.

http://www.fesabid.org/zaragoza2009/actas-fesabid2009/307-314.pdf

Diccionario de la Lengua Española. 22a ed. Madrid: Real Academia Española, 2001.

http://buscon.rae.es/drael/SrvltConsulta?TIPO_BUS=3\& LEMA=regla

Digital Curation Centre, Digital Preservation Europe (February 2007). DCC and DPE digital repository audit method based on risk assessment, v. 1, n. 0.

http://www.repositoryaudit.eu/download

Drambora (Digital Repository Audit Method: Based on Risk Assessment). Draft for public testing \& comment. Version 1.0. Digital Curation Centre (DCC): Digital Preservation Europe (DPE), 2007.

Duranti, Luciana. "From digital diplomatics to digital records forensics". Archivaria, 2009, n. 68, Fall, pp. 39-66.

Duranti, Luciana; Thibodeau, Kenneth. "The concept of record in interactive, experiential and dynamic environments: the view of InterPARES" Archival science, 2006, n. 1, March, pp. 13-68.

Fernández, Ricardo. "Una red vendió cientos de informes con datos reservados obtenidos de empleo". Laverdad.es, 1 marzo 2011.

http://www.laverdad.es/murcia/v/20110301/region/ven dio-cientos-informes-datos-20110301.html 
Foley, John. "Obama's cloud computing strategy takes shape". Information Week, May 11, 2009.

http://www.informationweek.com/blog/main/archives/ 2009/05/obamas_cloud_co.html;jsessionid=O2MVN3T3BR AV3QE1GHRSKHWATMY32JVN

Foucault, Michel. La arqueología del saber. Buenos Aires: Siglo XXI, 2005.

Foucault, Michel. El orden del discurso. Barcelona: Tusquets, 1999.

Furner, Jonathan. "Análisis conceptual: un método para comprender la información como evidencia y la evidencia como información". En: Gilliland, Anne; McKemmish, Sue: Nuevos métodos de investigación en archivística. Cartagena: Ayuntamiento: 3000 Informática, 2006, pp. 99-133.

Gellman, Robert. Privacy in the clouds: risks to privacy and confidentiality from cloud computing. World Privacy Forum, 2009.

Gilder, George. "The information factories". Wired, 2006, n. 14.10, October.

http://www.wired.com/wired/archive/14.10/cloudware. html

Gilliland-Swetland, Anne. Enduring paradigm, new opportunities: the value of the archival perspective in the digital environment. Washington DC: Council on Library and Information Resources, 2000.

http://www.clir.org/pubs/reports/pub89/pub89.pdf

Harvey, Ross. "La conservación de nuestro patrimonio digital: estrategias de conversión y migración". En: Seminario internacional o futuro da memoria: o patrimonio arquivístico dixital. Santiago de Compostela. 18-19 de nov. de 2010.

Heslop, Helen; Davis, Simon; Wilson, Andrew. An approach to the preservation of digital records. Canberra: National Archives, 2002.

http://naa.gov.au/Images/An-approach-Green-Paper tcm2-888.pdf

Hurley, Chris. Relationships in records, 2001-2004. http://www.sims.monash.edu.au/research/rcrg/publica tions/relationships-in-records-rev-3b.rtf

lacovino, Livia. "Beyond distributed networks: participatory governance of digital memory". En: Seminario internacional o futuro da memoria: o patrimonio arquivístico dixital. Santiago de Compostela, 18-19 de nov. de 2010.

lacovino, Livia. "Gestión de registros y gobernación jurídica". En: McKemmish, Sue; Piggott, Michael; Reed, Barbara; Upward, Frank: Archivos: gestión de registros en sociedad. Cartagena: Ayuntamiento: 3000 Informática, 2007.

InterPARES 2 Terminology database.

http://www.interpares.org/ip2/ip2_terminology_db.cfm

ISO/TR 18492:2005: Long-term preservation of electronic document-based information. Geneva: Organización Internacional de Normalización, 2005.

Ketelaar, Eric. "Archivalization and archiving". Archives and manuscripts, 1999, n. 27, pp. 54-61.

Ketelaar, Eric. Los archivos inmersos en el futuro. En: Se- minario internacional o futuro da memoria: o patrimonio arquivístico dixital. Santiago de Compostela. 18-19 de nov. de 2010.

Ketelaar, Eric. "Gestión de registros y poder social". En: McKemmish, Sue; Piggott, Michael; Reed, Barbara; Upward, Frank (eds.): Archivos: gestión de registros en sociedad. Cartagena: Ayuntamiento: 3000 Informática, 2007.

Ketelaar, Eric. "Writing on archiving machines". En: Neef, Sonja; van Dijck, José; Ketelaar, Eric (eds.): Sign here! Handwriting in the age of new media. Amsterdam: Amsterdam University Press, 2006, pp. 183-195.

McKemmish, Sue. "Evidencia de mí, evidencia de nosotros... en un espacio digital". En: VI Jornades de l'Associació d'Arxivers i Gestors de Documents Valencians: la e-archivística en la e-administració. Valencia, 5-7 de mayo de 2010.

McKemmish, Sue. "Placing records continuum theory and practice". En: Archival science, 2001, v. 1, n. 4, December, pp. 333-359.

McKemmish, Sue. "Trazas: documento, registro, archivo, archivos". En: McKemmish, Sue; Piggott, Michael; Reed, Barbara; Upward, Frank: Archivos: gestión de registros en sociedad. Cartagena: Ayuntamiento: 3000 Informática, 2007, pp. 21-46.

Mell, Peter; Grance, Tim. The NIST definition of cloud computing. National Institute of Standards and Technology, 2009.

http://csrc.nist.gov/groups/SNS/cloud-computing

Nesmith, Tom. "Seeing archives: postmodernism and the changing intellectual place of archives". The American archivist, 2002, v. 65, n. 1, Spring/Summer, pp. 24-41.

Nesmith, Tom. "Still fuzzy, but more accurate: some thoughts on the 'ghosts' of archival theory". Archivaria, 1999, n. 47, Spring, pp. 136-150.

Reed, Barbara. "Registros". En: McKemmish, Sue; Piggott, Michael; Reed, Barbara; Upward, Frank: Archivos: gestión de registros en sociedad. Cartagena: Ayuntamiento: 3000 Informática, 2007, pp. 169-173.

Reed, Barbara. "Service oriented architectures and recordkeeping". Records management journal, 2008, v. 18, n. 1, pp. 7-20.

Ross, Seamus; Hedstrom, Margaret. "Preservation research and sustainable digital libraries". International journal of digital libraries, 2005, v. 5, n. 4, pp. 317-325.

http://eprints.ERPANET.org/archive/00000095

Rothenberg, Jeff. "Preserving authentic digital information". En: Authenticity in a digital environment. Washington, DC: Council on Library and Information Resources, 2000, pp. 51-68.

http://www.clir.org/pubs/reports/pub92/rothenberg.html

Samouelian, Mary. "Embracing web 2.0: archives and the newest generation of web applications". The American archivist, 2009, v. 72, n. 1, Spring/Summer, pp. 42-70.

Schauder, Don; Stillman, Larry; Johanson, Graeme. "Sustaining a community network: the information continuum, 
e-democracy and the case of Vicnet". The journal of community informatics, 2005, v. 1, n. 2, pp. 79-102.

Schauder, Don; Stillman, Larry; Johanson, Graeme. "Sustaining and transforming a community network: the information continuum model and the case of Vicnet". En: CIRN 2004 Colloquium and conference, Prato, Italy, 29 Sept-1 Oct 2004. http://www.ccnr.net/?q=node/99

Spínola, Maria. An essential guide to possibilities and risks of cloud computing. June 2009.

http://www.mariaspinola.com/cloud-computing/

Thibodeau, Kenneth. "Preserving digital memory at the Na- tional Archives and Records Administration of the US". En: Workshop on conservation of digital memories. Second national conference on archives, Bologna, Italy. 20 Nov 2009, pp. 1-9.

Twining, William. "Evidence as a multi-disciplinary subject". En: Conf on inference, culture and ordinary thinking in dispute resolution, 2003, pp. 1-18.

Zonificación agro-ecológica: guía general. Roma: Organización de las Naciones Unidas para la Agricultura y la Alimentación, 1997.

http://www.fao.org/docrep/W2962S/W2962S00.htm

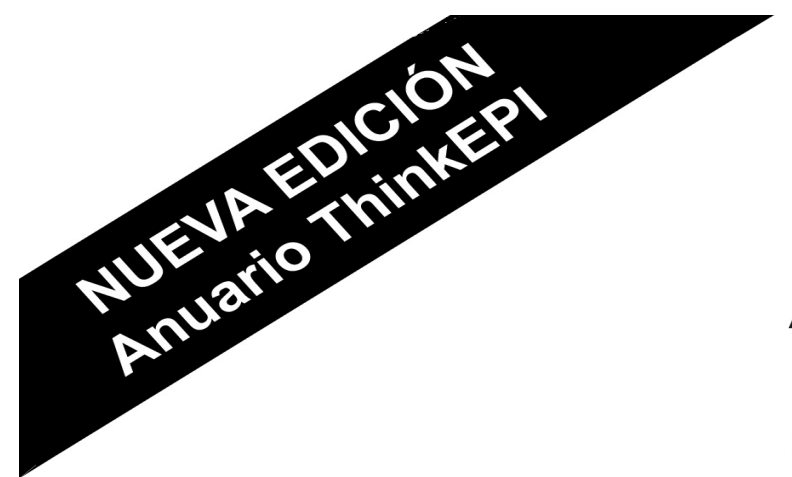

\section{1 Análisis de tendencias en información y documentación}

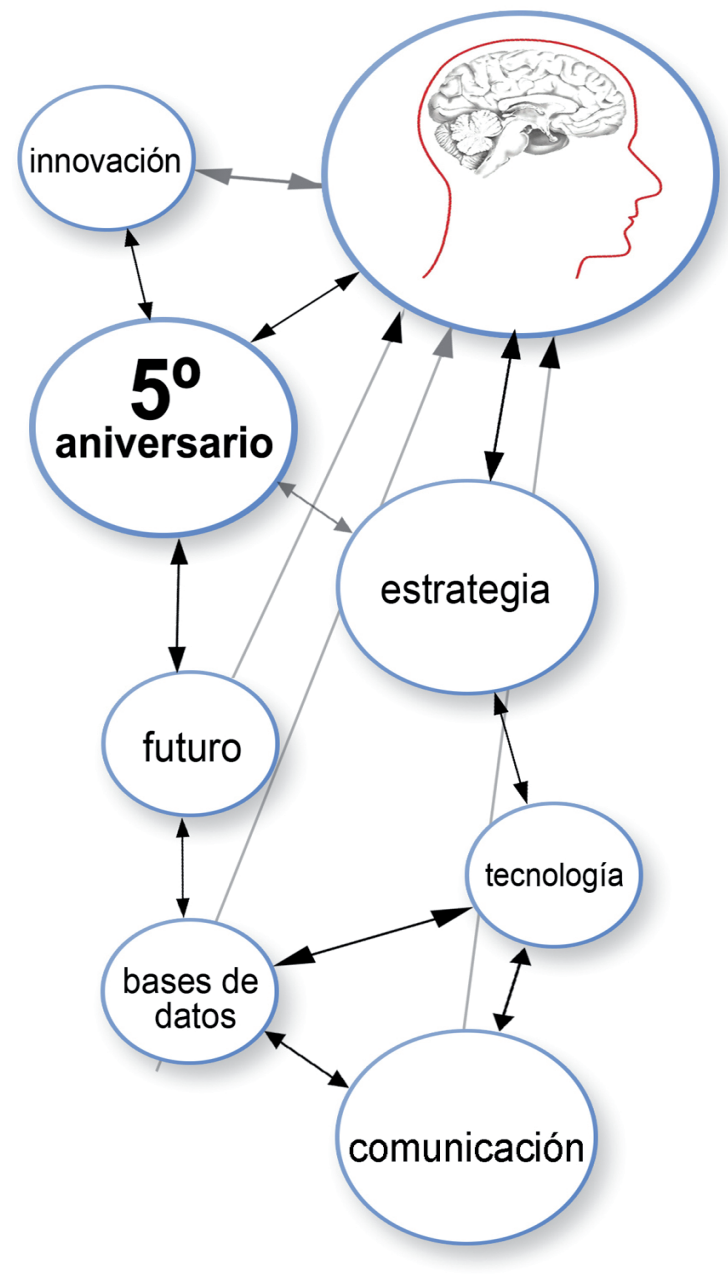

ANUARIO

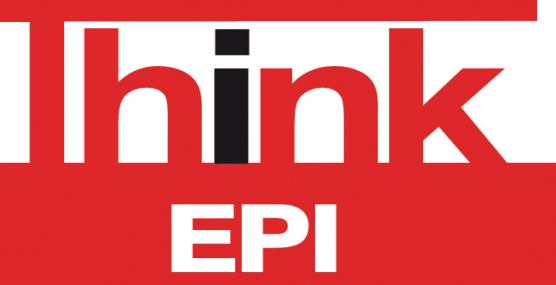

Algunos de los títulos:

Especialistas en información y documentación: ¿avanzamos algo? Carlota Bustelo-Ruesta

Facetas profesionales en documentación audiovisual Eugenio López-de-Quintana

De las TIC a las TAC: tecnologías del aprendizaje y del conocimiento

Roser Lozano

Integrados en la investigación: los embedded librarians Daniel Torres-Salinas

Profesionales de la información y compromiso con el desarrollo José-Antonio Moreiro

\section{Ya puedes pasarnos tu pedido:}

\section{http://www.thinkepi.net/anuario.html}

$\begin{array}{lc}\text { Anuario ThinkEPI } 2011 & 90,38+\text { IVA }=94 € \\ \text { Anuario ThinkEPI } 2010 & 46,15+\text { IVA }=48 € \\ \text { Anuario ThinkEPI } 2009 & 28,85+\text { IVA }=30 € \\ \text { Anuario ThinkEPI } 2011+2010 & 129,81+\text { IVA }=135 € \\ \text { Anuario ThinkEPI } 2010+2009 & 71,15+\text { IVA }=74 € \\ \text { Anuario ThinkEPI } 2011+2010+2009 & 163,46+\text { IVA }=170 €\end{array}$

Anuario ThinkEPI 2011

$90,38+$ IVA $=94 €$

$46,15+$ IVA $=48 €$

$28,85+$ IVA $=30 €$

$29,81+\mathrm{IVA}=135 €$

$163,46+$ IVA $=170 €$ 